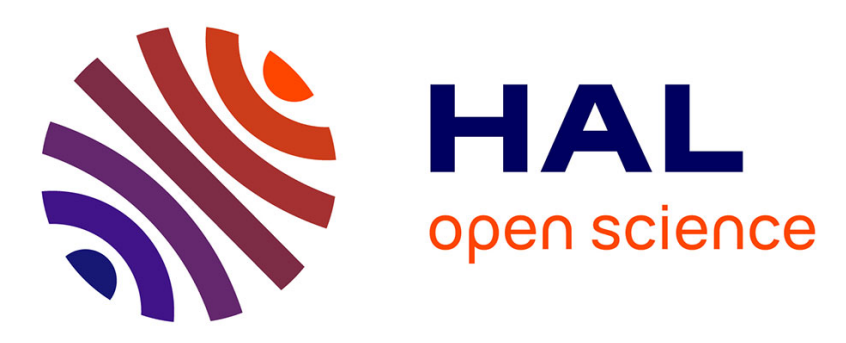

\title{
Caractérisation des états d'interface dans des transistors MOS submicroniques par différentes techniques de pompage de charge
}

Jean-Luc Autran, Frédéric Seigneur, Jacques Delmas, Carole Plossu, Bernard Balland

\section{To cite this version:}

Jean-Luc Autran, Frédéric Seigneur, Jacques Delmas, Carole Plossu, Bernard Balland. Caractérisation des états d'interface dans des transistors MOS submicroniques par différentes techniques de pompage de charge. Journal de Physique III, 1993, 3 (10), pp.1947-1961. 10.1051/jp3:1993252 . jpa-00249057

\section{HAL Id: jpa-00249057 https://hal.science/jpa-00249057}

Submitted on 1 Jan 1993

HAL is a multi-disciplinary open access archive for the deposit and dissemination of scientific research documents, whether they are published or not. The documents may come from teaching and research institutions in France or abroad, or from public or private research centers.
L'archive ouverte pluridisciplinaire $\mathbf{H A L}$, est destinée au dépôt et à la diffusion de documents scientifiques de niveau recherche, publiés ou non, émanant des établissements d'enseignement et de recherche français ou étrangers, des laboratoires publics ou privés. 


\title{
Caractérisation des états d'interface dans des transistors MOS submicroniques par différentes techniques de pompage de charge $(*)$
}

\author{
Jean-Luc Autran, Frédéric Seigneur, Jacques Delmas, Carole Plossu et Bernard Balland \\ Laboratoire de Physique de la Matière, Associé au Centre National de la Recherche Scientifique, \\ URA n 358, Institut Natıonal des Sciences Appliquées de Lyon, 20 avenue Albert Einstein, \\ 69621 Villeurbanne Cedex, France
}

(Reçu le 4 mars 1993, révisé le 7 juillet 1993, accepté le 13 juillet 1993)

\begin{abstract}
Résumé. - Nous avons effectué une étude comparative des différentes techniques de pompage de charge (classique, à trois niveaux, spectroscopique) et d'analyse électrique conventionnelles (DLTS, mesures C-V) sur des structures MOS et MOSFET submicroniques. La répartition énergétique de la densité d'états d'interface a été déterminée pour des substrats d'orientation $\langle 100\rangle$ de type $\mathrm{N}$ et $\mathrm{P}$ à différentes étapes d'un procédé de fabrication CMOS. Nous avons ainsi montré que les techniques de pompage de charge sont de puissants outils de caractérisation et de diagnostic permettant d'évaluer, avec une grande sensibilité, l'influence des traitements thermiques de passivation et des incidents de procédé sur la qualité interfaciale des dispositifs.

Abstract. - We have made a comparative study between different charge pumping techniques (standard, three-level, spectroscopic) and conventional electrical measurements (DLTS, C-V) on submicrometer MOSFET's and MOS devices. The energy distribution of interface states density has been determined for $\mathrm{N}$ and $\mathrm{P}$ type $\langle 100\rangle$ substrates at different stages of a CMOS process. We have shown that charge pumping techniques are powerful tools for characterizaton and diagnostic which allow to evaluate, with a great sensitivity, the influence of passivation annealings and process accidents on the quality of $\mathrm{Si}-\mathrm{SiO}_{2}$ interface.
\end{abstract}

\section{Introduction.}

Au fur et à mesure que diminuent les dimensions des composants électroniques intégrés, de nouvelles techniques de caractérisation s'avèrent nécessaires pour étudier les causes d'instabilité ou de vieillissement de ces dispositifs. La découverte du phénomène de pompage de charge dans les transistors MOS [1] a permis le développement, au cours des dix dernières années,

$\left(^{*}\right)$ Etude réalisée en collaboration avec le laboratoire Failure Analysis de l'usine IBM France de Corbell-Essonnes. 
d'une vaste panoplie de techniques particulièrement bien adaptées aux nouvelles dimensions des structures [2-17]. Il s'est avéré que son champ d'investigation a largement dépassé celui des techniques conventionnelles sur capacité MOS, telles que les mesures capacitives ou de conductance. Ceci est directement lié aux propriétés intrinsèques du composant, le transistor MOS, qui autorise, au niveau du canal, des courants de porteurs de charge minoritaires latéraux (vers ou depuis la source et le drain) et une modulation aisée de la zone électriquement active de l'interface (par modulation des zones de charge d'espace des jonctions source/substrat et drain/substrat via les polarisations appliquées). De telles possibilités ont été exploitées en pompage de charge classique $(2 \mathrm{CP})$, pour accéder à la répartition énergétique et spatiale des états électroniques à la surface du semiconducteur [2-11]. Quant à l'étude plus approfondie des différents régimes d'émission des porteurs de charge par les états d'interface, celle-ci a conduit au développement des récentes techniques de pompage de charge, dont l'application à des transistors submicroniques est une des particularités de notre travail. Dans le pompage de charge à trois niveaux (3CP), la durée du régime d'émission et la position du niveau de Fermi sont déterminées par l'introduction d'un troisième niveau de tension de grille [12-20] alors que dans le cas du pompage de charge spectroscopique (SCP), ce sont la température et les temps de transition du signal de grille qui jouent ce rôle [21, 22]. Il a été montré en [14] puis en [16] la faisabilité de mesure en pompage trois niveaux sur des transistors à faible longueur de canal présentant des densités d'états d'interface peu élevées (de $10^{10}$ à quelques $10^{11} \mathrm{eV}^{-1} \cdot \mathrm{cm}^{-2}$ ). L'un des objectifs du présent travail a été d'étudier la répartition énergétique des états d'interface, sur des composants issus de différentes étapes d'un procédé industriel CMOS, par différentes techniques de pompage de charge et conventionnelles. Nous avons effectué, sur les mêmes dispositifs de test, des mesures en pompage de charge classique, à trois niveaux et spectroscopique. La présence de capacités, de surfaces suffisantes, à proximité immédiate des transistors testés nous a permis de réaliser en outre des mesures capacitives quasi statiques et hautes fréquences ainsi que des mesures de DLTS (Deep Level Transient Spectroscopy). Nous présentons dans cet article la première étude comparative de toutes ces techniques pour plusieurs lots de transistors à canal $\mathrm{N}$ et $\mathrm{P}$ qui nous a permis de préciser l'influence qualitative et quantitative du procédé d'élaboration des composants MOS sur les propriétés électriques de l'interface $\mathrm{Si}-\mathrm{SiO}_{2}$.

\section{Approche spectroscopique du pompage de charge.}

Dans la version proposée par Van den Bosch et al. [21, 22], le pompage de charge spectroscopique est une technique de pompage de charge classique à deux niveaux de tension [2] faisant intervenir le paramètre température. Il consiste à suivre l'évolution du courant pompé en fonction de la température, pour deux signaux de grille présentant des temps de montée $t_{\mathrm{r}}$ (respectivement de descente $t_{\mathrm{f}}$ ) différents, les autres paramètres des signaux étant identiques (Fig. 1a). La moitié inférieure (respectivement supérieure) de la bandè interdite est ainsi balayée par une fenêtre énergétique définie par $t_{\mathrm{r} 1}$ et $t_{\mathrm{r} 2}$ à $t_{\mathrm{f}}$ constant (respectivement $t_{\mathrm{f} 1}$ et $t_{\mathrm{f} 2}$ à $t_{\mathrm{r}}$ constant) se déplaçant sur l'échelle des énergies sous l'action de la rampe de température (Fig. 1b).

La charge pompée au niveau du substrat, par période du signal de grille et par unité d'aire, a pour expression la plus générale :

$$
Q_{\mathrm{cp}}\left(t_{\mathrm{r}}, t_{\mathrm{f}}\right)=q \int_{E_{\mathrm{cm}, \mathrm{h}}\left(t_{\mathrm{r}}\right)}^{E_{\mathrm{em} \mathrm{e}}\left(t_{\mathrm{f}}\right)} D_{\mathrm{tt}}(E) \mathrm{d} E
$$

où $E_{\mathrm{em}, \mathrm{h}}\left(t_{\mathrm{r}}\right)$ (respectivement $E_{\mathrm{em}, \mathrm{e}}\left(t_{\mathrm{f}}\right)$ ) est la position du niveau de Fermi à la fin du processus d'émission hors équilibre de trous (respectivement d'électrons) par les états d'interface. Une 


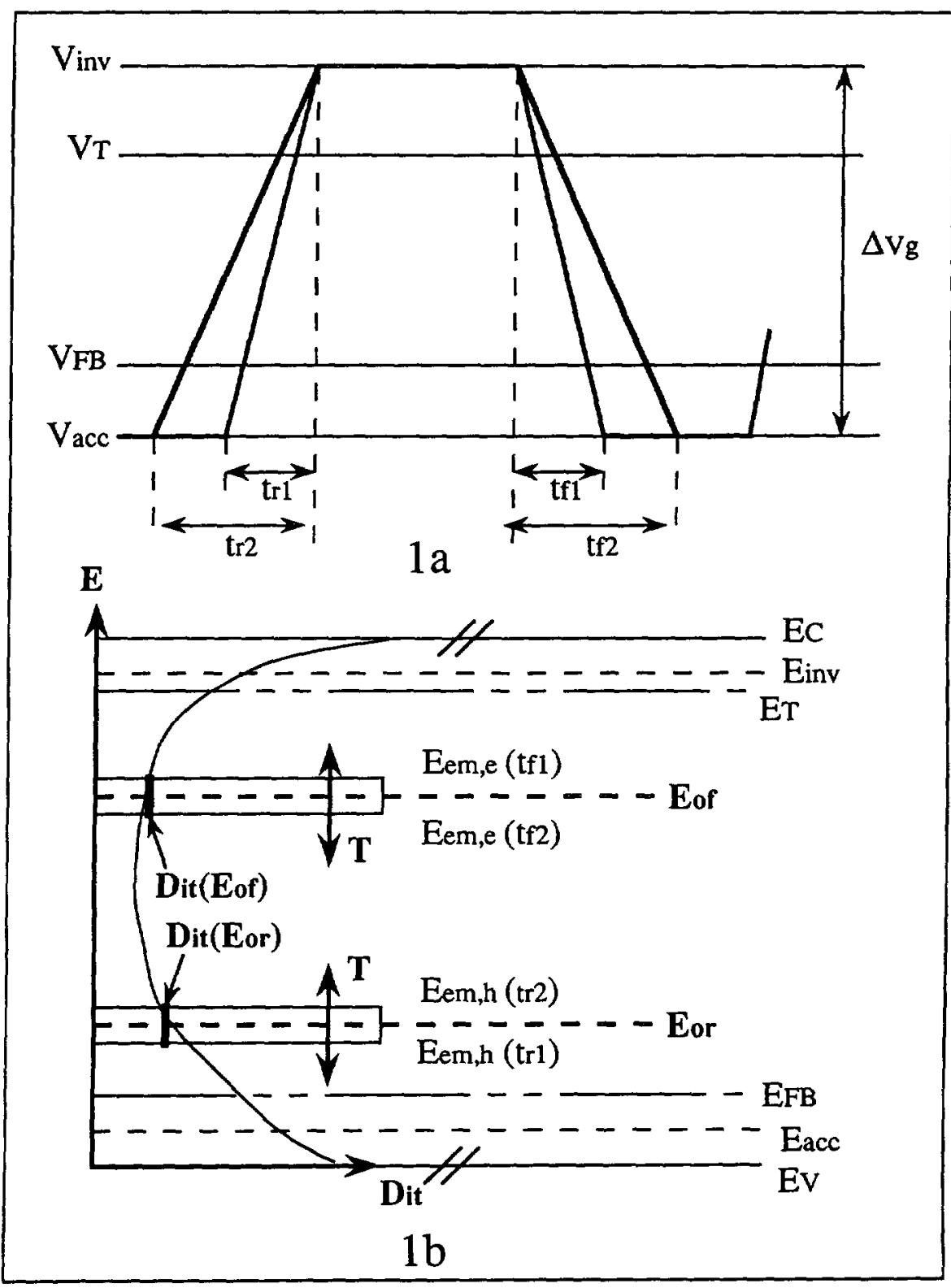

Fig. 1. - a) Signal de grille à deux niveaux de tension utilisé en pompage de charge spectroscopique. Une fenêtre d'émission est définie par le choix de 2 valeurs distinctes du temps de montée ou de descente, l'autre temps de transition du signal étant constant. b) Diagramme énergétique correspondant.

(a) Two-level gate voltage signal used in spectroscopic charge pumping. Emission window is defined by 2 distinct values of the fall or the rise time, the other transition time of the signal being fixed. b) Energy diagram corresponding to a).] 
analyse détaillée de ces processus d'émission hors équilibre des porteurs de charge par les états rapides de l"interface conduit aux expressions suivantes, valables pour des temps $t_{\mathrm{r}}$ et $t_{\mathrm{f}}$ supérieurs à $10^{-7} \mathrm{~s}[22]$ :

$$
\begin{aligned}
& E_{\mathrm{em}, \mathrm{h}}\left(t_{\mathrm{r}}\right)=E_{\mathrm{t}}+k T \ln \left[\sigma_{\mathrm{h}} v_{\mathrm{th}, \mathrm{h}} n_{1} \frac{V_{\mathrm{T}}-V_{\mathrm{FB}}}{\Delta V_{\mathrm{G}}} t_{\mathrm{r}}\right] \\
& E_{\mathrm{em}, \mathrm{e}}\left(t_{\mathrm{f}}\right)=E_{1}+k T \ln \left[\sigma_{\mathrm{e}} v_{\mathrm{th}, \mathrm{e}} n_{1} \frac{V_{\mathrm{T}}-V_{\mathrm{FB}}}{\Delta V_{\mathrm{G}}} t_{\mathrm{f}}\right]
\end{aligned}
$$

où $E_{1}$ est le niveau d'énergie intrinsèque, $T$ la température en Kelvin, $n_{1}$ la concentration intrinsèque des porteurs de charge, $\sigma_{\mathrm{e}}$ et $\sigma_{\mathrm{h}}$ les sections efficaces de capture pour les électrons et les trous, $v_{\text {th, e }}$ et $v_{\text {th. h }}$ leur vitesse thermique respective, $V_{\mathrm{FB}}$ la tension de bande plate du transistor, $V_{\mathrm{T}}$ sa tension de seuil et $\Delta V_{\mathrm{G}}$ la hauteur crête-à-crête du pulse appliqué sur la grille. Si $f$ est la fréquence du signal de grille et $A_{G}$ l'aire effective du canal, l'expression du courant pompé au niveau du substrat s'écrit :

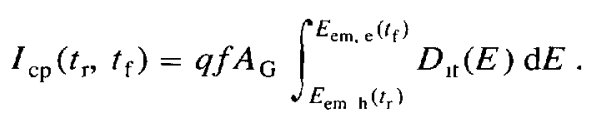

Cette expression dépend fortement de la température puisque les niveaux $E_{\mathrm{em}, \mathrm{h}}\left(t_{\mathrm{r}}\right)$ et $E_{\text {em, e }}\left(t_{\mathrm{f}}\right)$ sont directement dépendants de $T$. A noter que l'aire effective de canal $A_{\mathrm{G}}$ dépend également, en toute rigueur, de la température, puisque l'étendue des zones de charge d'espace des jonctions source/substrat et drain/substrat est fonction de $T$. On appelle signal spectroscopique correspondant à la fenêtre d'énergie déterminée par $t_{\mathrm{r} 1}$ et $t_{\mathrm{r} 2}$ à $t_{\mathrm{f}}$ constant la quantité $S_{\mathrm{r}}\left(t_{\mathrm{r} 1}, t_{\mathrm{r} 2}\right)=I_{\mathrm{cp}}\left(t_{\mathrm{r} 1}, t_{\mathrm{f}}\right)-I_{\mathrm{cp}}\left(t_{\mathrm{r} 2}, t_{\mathrm{f}}\right)$. D'après (4), elle a donc pour expression :

$$
S_{\mathrm{r}}\left(t_{\mathrm{r} 1}, t_{\mathrm{r} 2}\right)=q f A_{\mathrm{G}} \int_{E_{\mathrm{em} \mathrm{h}}\left(t_{\mathrm{r} 2}\right)}^{E_{\mathrm{em}, \mathrm{h}}\left(t_{\mathrm{r} 1}\right)} D_{\mathrm{tt}}(E) \mathrm{d} E .
$$

Soit $D_{\mathrm{tt}}\left(E_{\mathrm{or}}\right)$ la densité moyenne d'états d'interface dans la fenêtre d'énergie moyenne $E_{\text {or }}$ définie par $\left(t_{\mathrm{r} 1}, t_{\mathrm{r} 2}\right)$. D’après (2), cette énergie moyenne vaut :

$$
E_{\text {or }}=E_{1}+k T \ln \left[\sigma_{\mathrm{h}} v_{\mathrm{th}, \mathrm{h}} n_{1} \frac{V_{\mathrm{T}}-V_{\mathrm{FB}}}{\Delta V_{\mathrm{G}}} \frac{t_{\mathrm{r} 1}+t_{\mathrm{r} 2}}{2}\right] \text {. }
$$

L'expression (5) se réduit alors à :

$$
S_{\mathrm{r}}\left(t_{\mathrm{r} 1}, t_{\mathrm{r} 2}\right)=q f A_{\mathrm{G}} D_{\mathrm{It}}\left(E_{\mathrm{or}}\right)\left[E_{\mathrm{em}, \mathrm{h}}\left(t_{\mathrm{r} 2}\right)-E_{\mathrm{em}, \mathrm{h}}\left(t_{\mathrm{r} 1}\right)\right]
$$

qui peut s'écrire, compte tenu de (2):

$$
S_{\mathrm{r}}\left(t_{\mathrm{r} 1}, t_{\mathrm{r} 2}\right)=q f A_{\mathrm{G}} D_{\mathrm{tt}}\left(E_{\mathrm{or}}\right) k T \ln \left(\frac{t_{\mathrm{r} 2}}{t_{\mathrm{r} 1}}\right) .
$$

De la même manière, on appelle signal spectroscopique correspondant à la fenêtre d"énergie moyenne $E_{\mathrm{of}}$ déterminée par $t_{\mathrm{f} 1}$ et $t_{\mathrm{ff} 2}$ à $t_{\mathrm{r}}$ constant la quantité $S_{\mathrm{f}}\left(t_{\mathrm{f} 1}, t_{\mathrm{f} 2}\right)=I_{\mathrm{cp}}\left(t_{\mathrm{r}}, t_{\mathrm{f} 1}\right)-$ $I_{\mathrm{cp}}\left(t_{\mathrm{r}}, t_{\mathrm{f} 2}\right)$ qui a pour expression, compte tenu de (3):

$$
S_{\mathrm{f}}\left(t_{\mathrm{f} 1}, t_{\mathrm{f} 2}\right)=q f A_{\mathrm{G}} D_{\mathrm{tt}}\left(E_{\mathrm{of}}\right) k T \ln \left(\frac{t_{\mathrm{t} 2}}{t_{\mathrm{t} 1}}\right) .
$$


L'énergie moyenne de cette fenêtre a pour expression. d'après (3) :

$$
E_{\mathrm{of}}=E_{\mathrm{t}}-k T \ln \left[\sigma_{\mathrm{e}} v_{\mathrm{th}, \mathrm{e}} n_{\mathrm{s}} \frac{V_{\mathrm{T}}-V_{\mathrm{FB}}}{\Delta V_{\mathrm{G}}} \frac{t_{\mathrm{f} 1}+t_{\mathrm{f} 2}}{2}\right] .
$$

Les quantités $\Delta E_{\mathrm{r}}=k T \ln \left(t_{\mathrm{r} 2} / t_{\mathrm{r} 1}\right)$ et $\Delta E_{\mathrm{f}}=k T \ln \left(t_{\mathrm{f} 2} / t_{\mathrm{f} 1}\right)$ représentent les largeurs des intervalles d'énergie qui contribuent aux signaux spectroscopiques. Ces derniers sont directement proportionnels respectivement à $D_{\mathrm{tt}}\left(\mathrm{E}_{\mathrm{or}}\right)$ et $D_{\mathrm{tt}}\left(E_{\mathrm{of}}\right)$. En faisant varier la température, il est donc possible de déterminer $D_{1 t}(E)$ sur la quasi totalité de la bande interdite à partir des signaux spectroscopiques, les positions énergétiques moyennes des fenêtres étant calculées à partir de (6) et (9) si l'on connaît les valeurs des sections efficaces de capture.

\section{Procédure expérimentale.}

3.1 STRUCTURES DE TEST. - - Les transistors et les capacités ont été fabriqués selon un procédé de technologie CMOS (longueur de canal dessinée $0,75 \mu \mathrm{m}$. épaisseur d'oxyde $14 \mathrm{~nm}$ ) dont les principaux paramètres technologiques ont été donnés en référence [16]. Les transistors utilisés ont une structure interdigitée : la source et le drain constituent une jonction unique en forme de «peigne » avec le substrat qui interpénètre le «peigne » de grille. Il en résulte un encombrement réduit offrant une aire de grille importante (environ $32000 \mu \mathrm{m}^{2}$ ) pour une faible longueur de canal. Les capacités ont, quant à elles, une surface suffisante (17000 $\mu^{2}$ ) pour permettre d'effectuer des mesures C-V et de DLTS d'états de surface. Ces différentes structures de test sont montées en boîtier pour permettre une étude en température, inhérente aux deux techniques spectroscopiques appliquées. Trois séries d'échantillons ont été étudiées pour des substrats de chaque type : la série $n^{\circ} 1$ correspond à des composants prélevés en ligne de production avant la toute première étape de recuit. La série $n 2$ est constituée d'échantillons ayant subi, par rapport à ceux de la série $n$, deux traitements thermiques de passivation sous hydrogène (une vingtaine de minutes à $400{ }^{\circ} \mathrm{C}$ chacun). Quant à la série $\mathrm{n} 3$, elle correspond à des composants mal passivés qui présentent, en test final, une qualité d'interface nettement inférieure à celle de la série 2, révélée par une déformation des courbes capacité-tension quasi statiques alors que la dérive des tensions de bande-plate est peu significative (inférieure à $0,05 \mathrm{~V}$ entre les séries 2 et 3). Il est apparu intéressant de caractériser de telles structures, en plus de celles des séries 1 et 2 . afin d'évaluer la réponse des différentes techniques dans un cas concret d'incident survenu lors d'une étape particulière du procédé.

3.2 Calcul Des SPECTRES. - Nous explicitons, dans ce paragraphe, la procédure de calcul des spectres de densité d'états d'interface que nous nous sommes fixée dans le cadre de cette étude. L'analyse débute par l'acquisition des caractéristiques $C-V$ haute fréquence (fréquence $1 \mathrm{MHz}$, amplitude du signal de mesure $40 \mathrm{mV}$ crête-à-crête) et quasi statique (rampe de polarisation de $20 \mathrm{mV} . \mathrm{s}^{-1}$ ) de la capacité. La relation entre le potentiel de surface $\Psi_{\mathrm{s}}$ et la tension de grille $V_{\mathrm{G}}$ est déduite de la courbe quasi statique par la méthode de Berglund [24]. Les spectres de la densité d'états d'interface sont calculés en utilisant la méthode C-V haute fréquence - basse fréquence à partir des courbes précédentes [23]. Des mesures de DLTS (Deep Level Transient Spectroscopy) [23] sont effectuées sur la même capacité. Les transitoires de capacité sont échantillonnés numériquement pour cinq couples de valeurs $\left(t_{1}, t_{2}\right)$ qui définissent cinq taux d'émission, lors d'une unique montée en température de 80 à $400 \mathrm{~K}$. La figure 2a montre les différentes courbes $\Delta C(T)=C\left(t_{2}\right)-C\left(t_{1}\right)$ obtenues pour la série $\mathrm{n} l$ de type $\mathrm{P}$ (le type du substrat indiqué dans les figures est toujours celui du texte). Pour ces structures de type $P$, les conditions de mesure sont les suivantes : tension de 

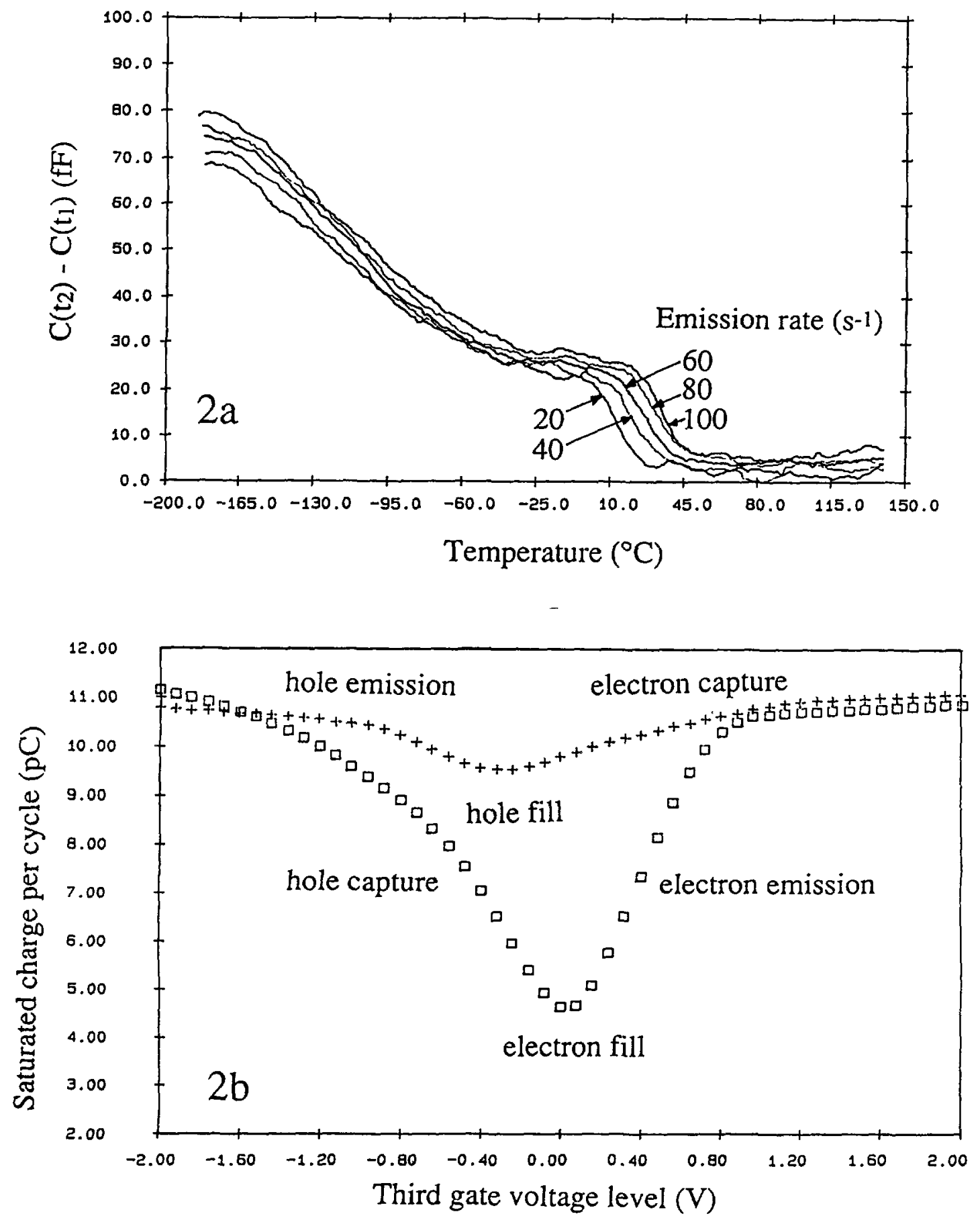

Fig. 2. - a) Signaux DLTS pour différents taux d'émission en fonction de la température. b) Charge pompée par cycle en régime de saturation en fonction de la tension de grille du troisième niveau de tension.

[a) DLTS signals for different emission rates as a function of temperature. b) Saturated charge pumped per cycle as a function of the third gate voltage level.] 
remplissage en accumulation $V_{\mathrm{A}}=-2,4 \mathrm{~V}$, durée du pulse de remplissage $t_{\mathrm{A}}=5 \mathrm{~ms}$, tension de déplétion $V_{\mathrm{D}}=-0,5 \mathrm{~V}$. Les spectres de densité d'états d'interface sont calculés à partir des différentes courbes $\Delta C(T)$ en prenant pour valeur de section efficace la valeur moyenne $\sigma$ obtenue par pompage de charge classique qui permet également de déterminer la densité d'états d'interface moyenne $\left\langle D_{11}\right\rangle[2,3]$. Le recouvrement des différents spectres (correspondant aux différentes vitesses d'émission) étant excellent, un seul spectre DLTS est représenté figure 4 ainsi que sur les autres figures. Par pompage de charge à trois niveaux sur transistor (durée du troisième niveau $10 \mathrm{~ms}$, période $12 \mathrm{~ms}$ ) [19], la répartition des états d'interface est calculée à partir de la caractéristique donnant la charge pompée en régime de saturation en fonction de la tension de grille du troisième niveau de tension, représentée figure $2 \mathrm{~b}$ pour la série $\mathrm{n}^{\circ} 1$ de type $\mathrm{P}$ (transistor $\mathrm{n}$-MOSFET). Le régime d'émission étant à l'équilibre thermodynamique, contrairement au régime de capture, seuls les points correspondant à l'émission d'électrons et de trous (Fig. 2b) sont pris en compte dans le calcul de la répartition énergétique des états [17]. On notera que la variation de la charge pompée en régime de saturation en fonction du troisième niveau de tension est inférieure dans le cas de l'émission de trous que dans le cas de l'émission d'électrons, tout simplement parce que dans le cas des structures de la série 1 (c'est également vrai pour les dispositifs de la série 2), la densité de pièges dans la moitié inférieure de la bande interdite est inférieure à celle de la moitié supérieure du gap. Enfin, nous réalisons l'acquisition des signaux $S_{\mathrm{r}}$ et $S_{\mathrm{f}}$ en pompage de charge spectroscopique pour différents couples de temps de montée et de descente du signal de grille. Les figures $3 a$ et $3 b$ récapitulent les différents signaux spectroscopiques obtenus pour un transistor de la série $n 1$ de type P. Comme dans le cas de la DLTS, nous prenons la valeur de la section efficace moyenne $\sigma$, obtenue par pompage de charge classique, pour calculer les spectres de densité d'états à partir des différents signaux spectroscopiques. Là encore, les différents spectres sont superposables, ce qui laisse supposer que le choix d'une valeur unique de section efficace de capture est une approximation valable. Cette procédure expérimentale est appliquée aux trois séries d'échantillons en prenant rigoureusement les mêmes paramètres temporels de mesure.

\section{Résultats et discussion.}

Nous récapitulons, dans ce paragraphe et dans les figures suivantes, l'ensemble des résultats que nous avons obtenus au cours de cette étude pour les séries 1 à 3 de type $N$ et $P$ du substrat (24 spectres typiques). Les spectres présentés sur une même figure ont été obtenus pour la même série et pour un même type de substrat. Les figures 4 à 6 représentent les différentes répartitions des états d'interface en fonction de l'énergie dans la bande interdite du silicium obtenues pour les séries $N$ et $P$ avant recuit (série $n^{\circ} 1$, Figs. 4 a et $4 b$ ), après recuit (série $n 2$, Figs. $5 a$ et $5 b$ ) et après un incident survenu lors du recuit (série $n^{\circ} 3$, Figs. $6 a$ et $6 b$ ). En outre, le tableau I indique les valeurs moyennes de la densité d'états d'interface et des sections efficaces de capture déterminées par pompage de charge classique.

4. 1 Comparaison Des TeCHNiques. - Pour les séries n 1 et 2, nous montrons que la densité d'états d'interface obtenue par mesures $\mathrm{C}-\mathrm{V}$ est toujours supérieure aux densités obtenues par $3 \mathrm{CP}$ et SCP. Un tel résultat vient confirmer la comparaison de Saks et Ancona concernant les positions relatives des spectres $3 \mathrm{CP}$ et $\mathrm{C}-\mathrm{V}$ [18]. En ce qui concerne la série $\mathrm{n} 3$ et contrairement aux autres séries, nous mettons en évidence une relative bonne correspondance des spectres obtenus par pompage de charge et mesures $\mathrm{C}-\mathrm{V}$. La comparaison entre spectres DLTS et spectres SCP ou 3CP montre un bon accord des distributions dans le cas des dispositifs sur substrat $\mathrm{N}$. En particulier, la figure $5 \mathrm{~b}$, correspondant à des structures de bonne qualité d'interface (après recuit), offre une comparaison entre DLTS et SCP semblable à celle 

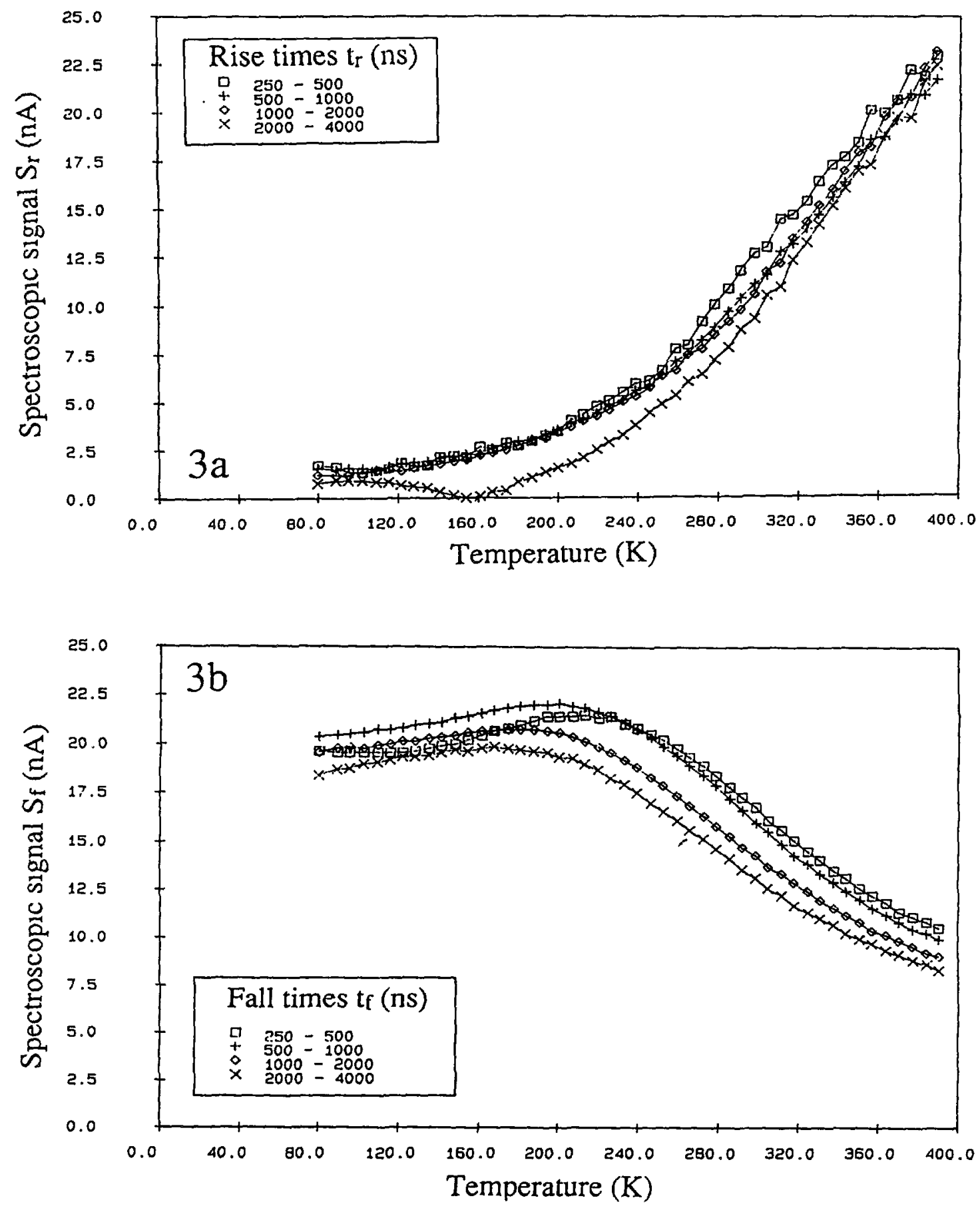

Fig. 3. - Signaux spectroscopiques pour différentes fenêtres d'émission définies par 2 valeurs distinctes du temps de montée $\left(t_{\mathrm{f}}=1 \mu \mathrm{s}\right.$ fixe) ou par 2 valeurs distinctes du temps de descente $\left(t_{\mathrm{r}}=1 \mu \mathrm{s}\right.$ fixe).

[Spectroscopic signals for different emission windows defined by 2 distinct values of the rise time (fixed $t_{\mathrm{f}}=1 \mu \mathrm{s}$ ) or by 2 distinct values of the fall time (fixed $t_{\mathrm{r}}=1 \mu \mathrm{s}$ ).] 

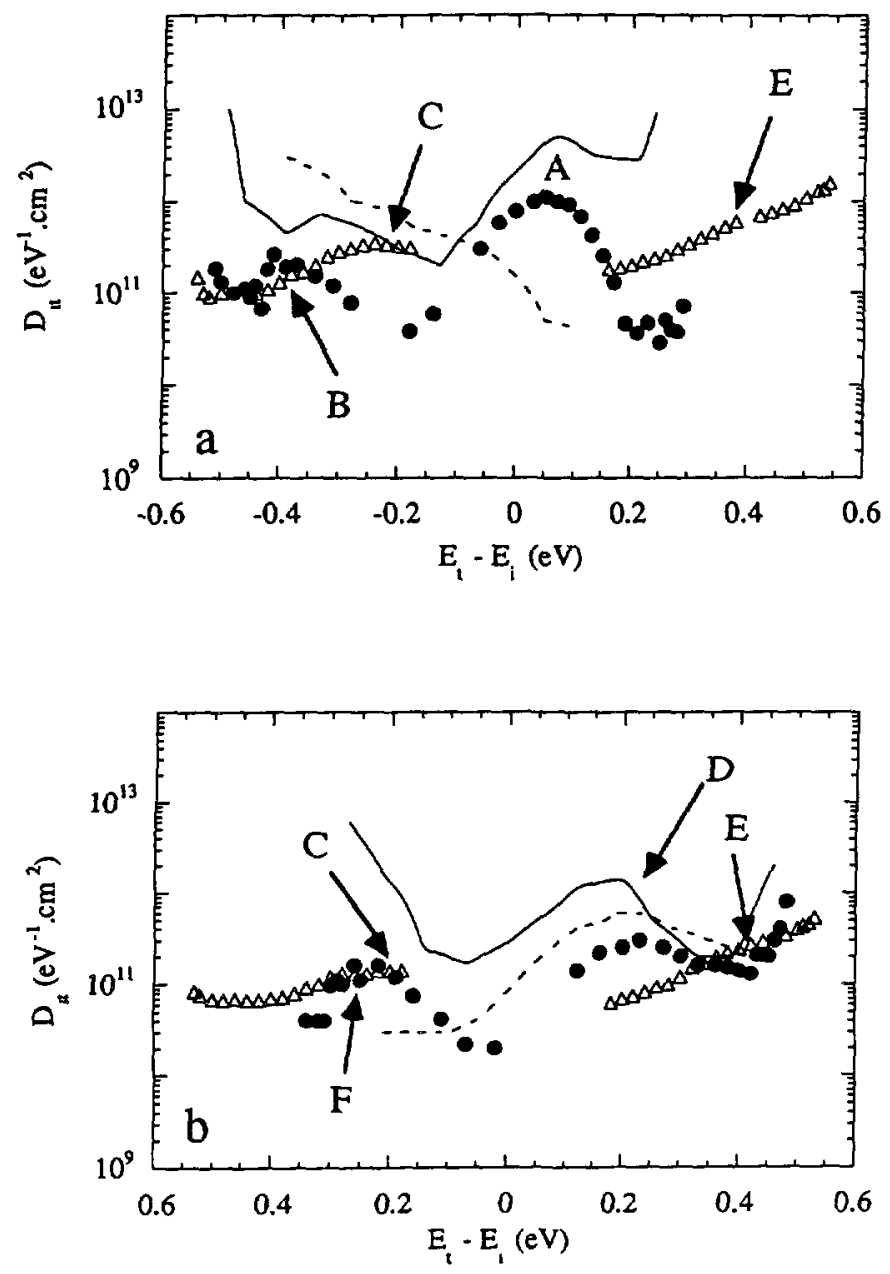

Fig. 4. - Spectres de la densité d'états d'interface dans la bande interdite du silicium obtenus par différentes techniques pour la série $\mathrm{n} 1$ de type $\mathrm{P}$ (a) et de type $\mathrm{N}$ (b). Ligne continue: mesures capacitives haute fréquence - quasi statique. Ligne pointillée: mesures DLTS. Points: pompage de charge à 3 niveaux. Triangles: pompage de charge spectroscopique.

[Energy distributions of interface state density in silicon bandgap corresponding to differents techniques for P-type (a) and N-type (b) series n 1. Full line: high frequency - quasi static capacitance measurements. Dotted line DLTS measurements. Dots : 3-level charge pumping. Triangles : spectroscopic charge pumping.]

publiée par Van den Bosh et al. [22]. Dans le cas des échantillons de type P, les spectres DLTS sont sensiblement au-dessus des autres courbes et divergent relativement par rapport aux autres courbes (Fig. 4a en particulier). D’une façon générale, nous constatons une assez grande cohérence des différents spectres pour la moitié de la bande interdite qui correspond à des pièges à porteurs majoritaires. Pour les pièges à porteurs minoritaires, dans la deuxième moitié de la bande interdite, on observe une relative divergence des courbes bien que l'allure générale des différents spectres soit toujours la même. Cette divergence a été rapportée par Saks et Ancona dans le cas de mesures $\mathrm{C}-\mathrm{V}$ et $3 \mathrm{CP}$ [18]. En ce qui concerne la position relative des 

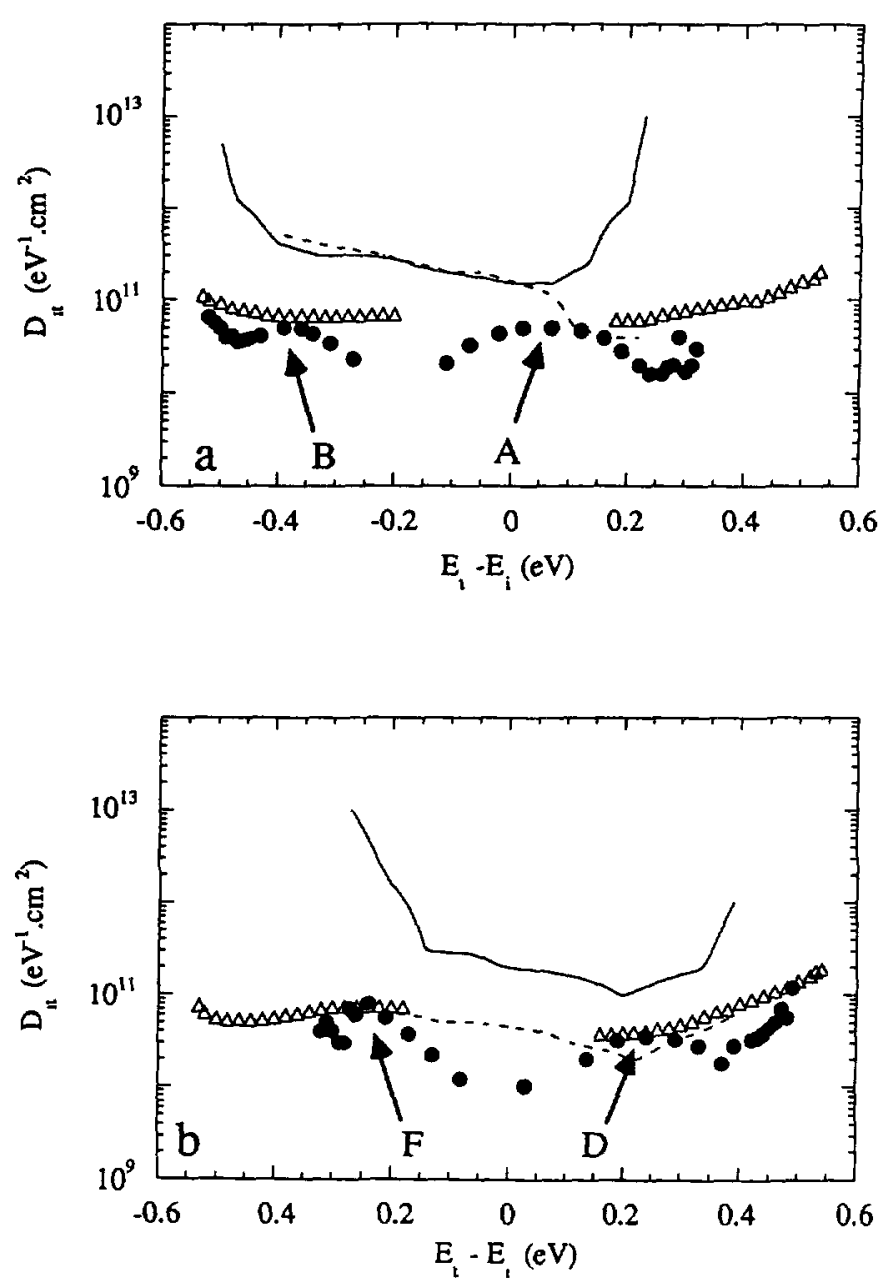

Fig. 5. - Spectres de la densité d'états d'interface dans la bande interdite du silicium obtenus par différentes techniques pour la série $\mathrm{n} 2$ de type $\mathrm{P}$ (a) et de type $\mathrm{N}$ (b). Ltgne continue: mesures capacitives haute fréquence - quasi statique. Ligne pointillée : mesures DLTS. Points . pompage de charge à 3 niveaux. Triangles: pompage de charge spectroscopique.

[Energy distributions of interface state density in silicon bandgap corresponding to differents techniques for P-type (a) and N-type (b) series n 2. Full line: high frequency - quasi static capacitance measurements. Dotted line : DLTS measurements. Dots $\cdot 3$-level charge pumping. Triangles : spectroscopic charge pumping.]

différents spectres, on peut penser que le caractère quasi statique de la mesure capacitive (faible vitesse de rampe de polarisation) fait intervenir un plus grand nombre d'états dans les spectres $\mathrm{C}-\mathrm{V}$ que les spectres de pompage, calculés à partir de signaux ne prenant en compte que la fraction des états d'interface ayant un temps d'émission inférieur à la durée d'émission fixée par le signal de grille. Autrement dit, le fait que les spectres $C-V$ soient en général audessus des spectres de pompage peut être interprété comme une sélectivité différente des états d'interface suivant la technique utilisée : les mesures $C-V$ apparaissent d'avantage sensibles à des états plus « lents » (temps d'émission longs) que les mesures en pompage de charge (temps 

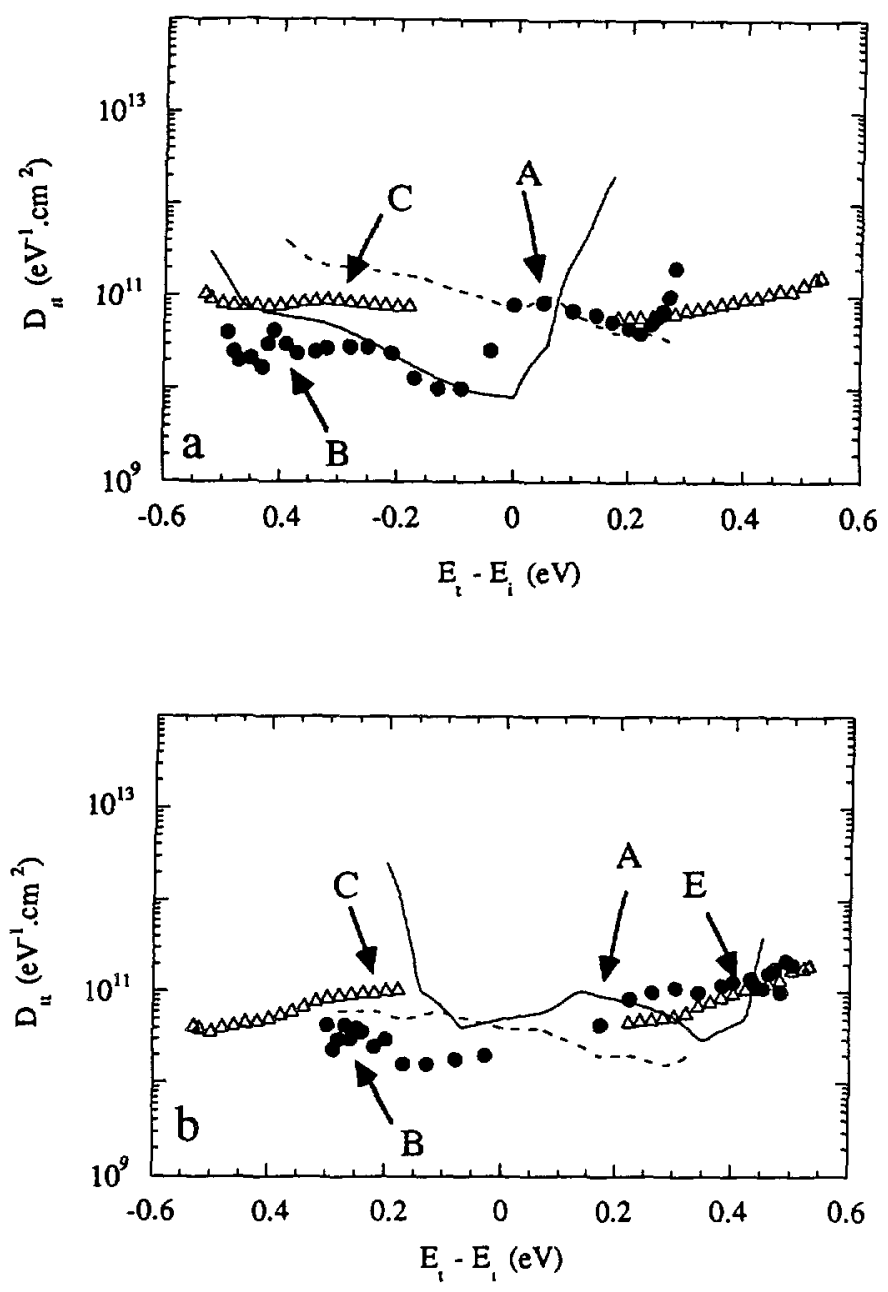

Fig. 6. - Spectres de la densité d'états d'interface dans la bande interdite du silicium obtenus par différentes techniques pour la série $\mathrm{n} 3$ de type $\mathrm{P}$ (a) et de type $\mathrm{N}$ (b). Ligne continue: mesures capacitives haute fréquence - quasi statique. Ligne pointillée : mesures DLTS. Points : pompage de charge à 3 niveaux. Triangles: pompage de charge spectroscopique.

[Energy distributions of interface state density in silicon bandgap corresponding to differents techniques for P-type (a) and N-type (b) series n 3. Full line: high frequency - quası static capacitance measurements. Dotted line : DLTS measurements. Dots : 3-level charge pumping. Triangles: spectroscopic charge pumping.]

d'émission inférieurs à $10 \mathrm{~ms}$ dans le cas du $3 \mathrm{CP}$ par exemple). Il en est de même pour les mesures DLTS [24] qui ont été réalisées pour des faibles taux d'émission (20 à $100 \mathrm{~s}^{-1}$ ) et qui impliquent un plus grand nombre d'états, dans la réponse électrique de la structure, que les mesures $3 \mathrm{CP}$ et $\mathrm{SCP}$. En particulier, pour cette dernière technique, et bien que la procédure d'analyse soit calquée sur celle de la DLTS, les taux d'émission ne sont pas du tout du même ordre de grandeur puisque l'on travaille avec des valeurs comprises entre $10^{4}$ et $10^{6} \mathrm{~s}^{-1}$ [22]. Seuls des états rapides participent donc à la réponse en pompage spectroscopique. Le cas de la série n 3 est intéressant car l'analyse de la position des différents spectres laisserait supposer 
Tableau I. - Valeurs moyennes de la densité d'états d'interface et des sections efficaces de capture obtenues par pompage de charge classique pour les différentes séries d'échantillons en fonction du type du substrat.

[Average values of interface state density and capture cross sections obtained by standard charge pumping for the differents series of devices as a function of the substrate type.]

\begin{tabular}{|c|c|c|}
\hline & P type & N type \\
\hline Series 1 & $\left\langle D_{\mathrm{tt}}\right\rangle=4,4 \times 10^{11} \mathrm{eV}^{-1} \cdot \mathrm{cm}^{-2}$ & $\left\langle D_{\mathrm{tt}}\right\rangle=2,0 \times 10^{11} \mathrm{eV}^{-1} \cdot \mathrm{cm}^{-2}$ \\
& $\langle\sigma\rangle=5 \times 10^{-15} \mathrm{~cm}^{2}$ & $\langle\sigma\rangle=6 \times 10^{-15} \mathrm{~cm}^{2}$ \\
\hline Series 2 & $\left\langle D_{\mathrm{tt}}\right\rangle=4,0 \times 10^{10} \mathrm{eV}^{-1} \cdot \mathrm{cm}^{-2}$ & $\left\langle D_{\mathrm{tt}}\right\rangle=3,8 \times 10^{10} \mathrm{eV}^{-1} \cdot \mathrm{cm}^{-2}$ \\
& $\langle\sigma\rangle=4 \times 10^{-15} \mathrm{~cm}^{2}$ & $\langle\sigma\rangle=5 \times 10^{-15} \mathrm{~cm}^{2}$ \\
\hline Series 3 & $\left\langle D_{\mathrm{tt}}\right\rangle=9,6 \times 10^{10} \mathrm{eV}^{-1} \cdot \mathrm{cm}^{-2}$ & $\left\langle D_{\mathrm{it}}\right\rangle=1,2 \times 10^{11} \mathrm{eV}^{-1} \cdot \mathrm{cm}^{-2}$ \\
& $\langle\sigma\rangle=5 \times 10^{-16} \mathrm{~cm}^{2}$ & $\langle\sigma\rangle=2 \times 10^{-16} \mathrm{~cm}^{2}$ \\
\hline
\end{tabular}

que la proportion d'états lents a été sensiblement modifiée par l'incident de procédé : bien que le nombre de pièges ait globalement augmenté, ceux-ci seraient plutôt des états rapides, détectés presque identiquement par les différentes techniques. La proportion d'états lents semblerait avoir diminué. A l'inverse, lors d'un recuit correctement effectué (série $\mathbf{n}^{\circ} 2$ ), le nombre d'états rapides diminuerait beaucoup plus que celui des états lents. En ce qui concerne la dispersion des spectres pour les pièges à minoritaires, nous pensons qu'il peut s'agir là du même problème de sélectivité auquel vient s'ajouter une erreur de mesure grandissante pour les techniques $C-V$ et DLTS. En effet, le modèle de calcul de la densité d'états d'interface devient approximatif à l'approche de l'inversion dans le cas de la méthode $\mathrm{C}-\mathrm{V}$ haute fréquence quasi statique [23]. Le problème est similaire en DLTS où les erreurs sur la densité d'états (à l'approche de la formation de la couche d'inversion) et sur la position énergétique (sections efficaces de capture supposées constantes) peuvent devenir non négligeables dans cette partie du spectre. A noter enfin que les valeurs moyennes de densité d'états d'interface obtenues par pompage de charge classique (Tab. I) sont en complet accord avec l'ensemble des spectres dans la partie du gap où ceux-ci sont cohérents entre eux et avec les spectres $3 \mathrm{CP}$ et SCP sur la totalité du gap.

4.2 INFLUENCE DU PROCÉDÉ. - Si l'on effectue un classement des trois séries d'échantillons suivant une densité d'états d'interface moyenne décroissante, on montre que toutes les techniques donnent strictement le même résultat, aussi bien pour les structures de type $\mathrm{N}$ que de type $P$. Toutes les structures de la série $n 1$ présentent. quelle que soit la technique de mesure utilisée, une densité d'états nettement supérieure à $10^{11} \mathrm{eV}^{-1} \cdot \mathrm{cm}^{-2}$ Celles de la série n 3 ont une densité d'états de l'ordre de $10^{11} \mathrm{eV}^{-1} \cdot \mathrm{cm}^{-2}$ et celles de la série n 2 sont caractérisées par une faible densité d'états, comprise entre $10^{10} \mathrm{eV}^{-1} \mathrm{~cm}^{-2}$ et $10^{11} \mathrm{eV}^{-1} \mathrm{~cm}^{-2}$ $\mathrm{Ce}$ classement est conforme à l'origine respective de chaque série d'échantillons (cf. Sect. 3.1). Une forte densité d'états d'interface est mesurée avant tout traitement thermique de passivation de l'interface (série $\mathrm{n} 1$ ). Cette densité d'état est considérablement réduite après deux recuits consécutifs sous hydrogène à $400^{\circ} \mathrm{C}$, comme le montrent les spectres des séries 1 et 2 . Les spectres de la série n 3 permettent de mettre clairement en évidence un incident au cours d'une phase particulière du procédé. D'une part, la densité d'états d'interface à certes diminué par rapport à celle de la série n 1 mais de façon moins significative que pour la série 
n 2. D'autre part. les spectres des séries n 2 et 3 présentent des différences d'allure notables, traduisant une anomalie au cours de l'un des traitements thermiques. Une étude plus approfondie des spectres de densité d'états d'interface fait apparaître un certain nombre de «pics» et de «bosses» de densité d'états dont certains apparaissent sur plusieurs spectres. Dans le cas des échantillons de type $\mathrm{P}$, trois pics sont mis en évidence à $E_{1}+0.07 \pm$ $0.02 \mathrm{eV}(\mathrm{A}), E_{1}-0.38 \pm 0.02 \mathrm{eV}$ (B) et $E_{1}-0.27 \pm 0.03 \mathrm{eV}(\mathrm{C})$. Il en est de même pour les échantillons de type $\mathrm{N}$ où l'on distingue trois pics à $E_{1}+0.22 \pm 0.04 \mathrm{eV}(\mathrm{D}), E_{1}+$ $0.37 \pm 0.02 \mathrm{eV}(\mathrm{E})$ et à $E_{\mathrm{I}}-0.24 \pm 0.02 \mathrm{eV}(\mathrm{F})$. Les pics $\mathrm{A}, \mathrm{B}, \mathrm{D}$ et $\mathrm{F}$ sont présents pour les trois séries d'échantillons. Seules leurs amplitudes respectives varient en fonction de la série, ce qui traduit une diminution du nombre des états qui leur sont associés après les étapes de recuit. Nous supposons qu'ils correspondent à la présence d'impuretés chimiques en surface du silicium inhérentes au procédé d'élaboration, ces impuretés pouvant diffuser ensuite au cours des recuits successifs dans le volume du semiconducteur. Les pics $\mathrm{C}$ et $\mathrm{E}$ sont moins marqués que les précédents et $n^{\circ}$ apparaissent que pour les séries $n^{\circ} 1$ et 3 . La figure 7 permet de suivre

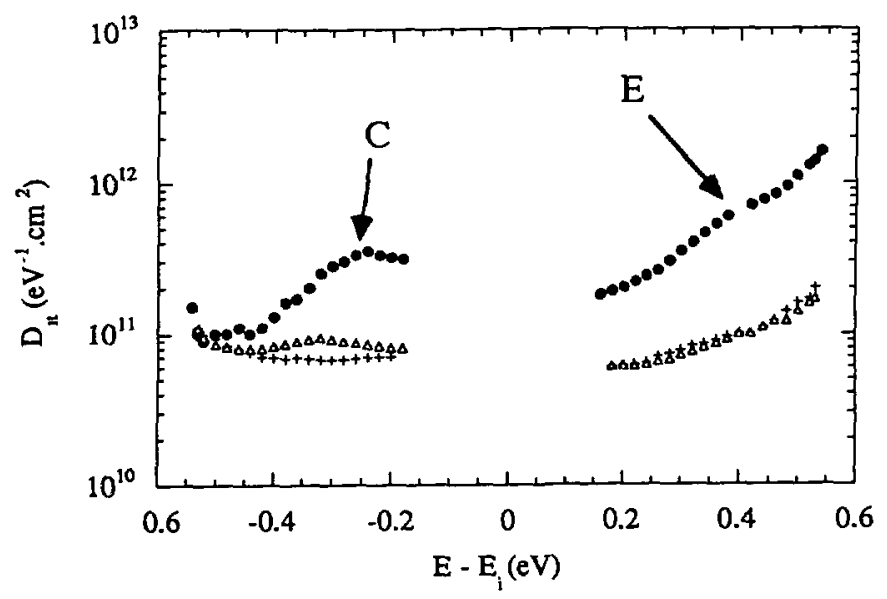

a)

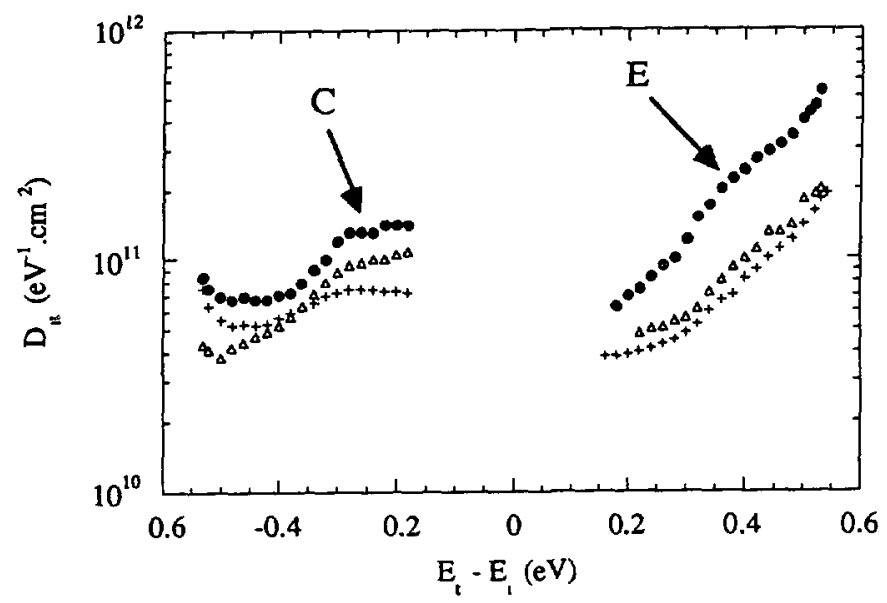

Fig. 7. - Spectres de la densité d'états d'interface dans la bande interdite du silicium de type P(a) et de type $\mathrm{N}(\mathrm{b})$ pour les séries $\mathrm{n} 1$ (points), 2 (croix) et 3 (triangles) obtenus par pompage de charge spectroscopique.

[Energy distributions of interface state density in silicon bandgap for P-type (a) and N-type (b) series $n 1$ (dots), 2 (cross) and 3 (triangles) obtained by spectroscopic charge pumping.] 
l'évolution de ces pics sur les distributions de pièges déterminées par pompage de charge spectroscopique en fonction de la série et du type des échantillons. Nous pensons que $\mathrm{C}$ et $\mathrm{E}$ pourraient correspondre à des pics associés à des défauts de l'interface dans la mesure où des analyses DLTS réalisées sur les jonctions source/substrat et drain/substrat des transistors n ont révélé la présence d'aucun défaut profond dans le volume du substrat. De plus, la forme particulière des signaux spectroscopiques associés à ces spectres montre que cette hypothèse est plausible. Par exemple, pour le spectre de la série n l de type P. la présence du pic E se traduit par une «bosse " élargie caractéristique sur les signaux de la figure $3 \mathrm{~b}$. Le pic $\mathrm{C}$ apparaissant en particulier sur le spectre de la série n 3 de type P (Fig. 7) alors qu'il n'apparaît pas sur le spectre de la série 2 de même type peut laisser supposer qu'il y a eu passivation partielle ou même dépassivation de certains états lors des étapes de recuit de la série $\mathrm{n}^{\circ} 3$. C'est une hypothèse qui est cependant difficile à vérifier tant que l'on ne peut intervenir directement au niveau du procédé d'élaboration pour favoriser la création de ces défauts. Néanmoins, la présence de ces deux pics permet d'apprécier la sensibilité des techniques de pompage de charge qui peuvent mettre en évidence des fluctuations de densité de quelques $10^{10} \mathrm{eV}^{-1} \mathrm{~cm}^{-2}$ sur un intervalle énergétique réduit $(0,2 \mathrm{eV})$ dans la bande interdite du silicium.

\section{Conclusion.}

Nous avons cherché à confronter les principales techniques de spectroscopie électrique des états de l'interface $\mathrm{Si}-\mathrm{SiO}_{2}\langle 100\rangle$ sur une grande variété de transistors et de capacités de relativement petites dimensions. Cette étude est la première analyse comparative systématique entre des techniques de pompage de charge et capacitives : elle concerne des structures de type $\mathrm{N}$ et de type $\mathrm{P}$ présentant des qualités interfaciales différentes. Nous avons utilisé la technique du pompage à trois niveaux, précédemment développée [2], et mis en œuvre une technique de pompage de charge spectroscopique basée sur une étude en température du courant pompé. Nous avons montré que les résultats obtenus par toutes ces techniques sont cohérents entre eux et que le recouvrement des spectres de densité d'états est excellent dans le cas des pièges à porteurs majoritaires. Ces méthodes de caractérisation nous ont ainsi permis de déterminer l'influence qualitative et quantitative d'un traitement thermique de passivation d'un procédé CMOS d'élaboration industriel sur les propriétés électriques de l'interface.

\section{Remerciements.}

Cette étude a pu être menée à bien grâce à la Compagnie IBM France. Nous tenons à remercier tout particulièrement le service 1878 de l'usine IBM France de Corbeil-Essonnes.

\section{Bibliographie}

[1] Brugler J. S., Jespers G. A., IEEE Trans. Electron Devices 16 (1969) 297.

[2] Groeseneken G., Maes H. E., Beltran N., De Keersmaecker R. F., IEEE Trans. Electron Devices 31 (1984) 42.

[3] Cilingiroglu U., Solid-State Electron. 28 (1985) 1127.

[4] Plossu C., Choquet C., Lubowiecki V., Balland B., Solid-State Commun. 65 (1988) 1231.

[5] Mahnkopf R., Przyrembel G., Wagemann H. G., J. Phys. Colloq. France 49 (1988), C4-775.

[6] Shaw J.-J., Wu K., IEDM Tech. Dig. (1989) 83.

[7] Saks N. S., Ancona M. G., IEEE Trans. Electron Devices 37 (1990) 1057.

[8] Henning A. K., Dimauro J. A., Electron. Lett. 27 (1991) 1445.

[9] Li X. M., Deen M. J., Solid-State Electron. 35 (1992) 1059. 
[10] Chen W., Ma T. P.. IEEE Electron Device Lett. 12 (1991) 393.

[11] Latyshev A. V., Lisovskii G. A., Lomako V. M., Sov. Technical Phys. Lett. 17 (1991) 710.

[12] Tseng W. L., J. Appl. Phys. 62 (1987) 591.

[13] Hofmann F., Krautschneider W. H., J. Appl. Phys. 65 (1989) 1358.

[14] Chung J. E., Muller R. S., Solid State Electron. 32 (1989) 867.

[15] Saks N. S., Ancona M. G., IEEE Electron Device Lett. 11 (1990) 339.

[16] Autran J. L., Balland B., Plossu C., Seigneur F., Gaborieau L. M., J. Phys. III France 3 (1993) 33.

[17] Ancona M. G., Saks N. S., J. Appl. Phys. 71 (1992) 4415.

[18] Saks N. S., Ancona M. G., Appl. Phys. Lett. 60 (1992) 2261.

[19] Autran J. L., Djahli F., Balland B., Plossu C., Gaborieau L. M., Solid State Commun. 84 (1992) 607.

[20] Kejhar M.. IEEE Electron Device Lett. 13 (1992) 344.

[21] Van den Bosch G., Groeseneken G., Heremans P., Maes H. E., ESSDERC 90, 20th European Solid State-Device Research Conference, p. 579.

[22] Van den Bosch G., Groeseneken G., Heremans P., Maes H. E., IEEE Trans. Electron Devices 38 (1991) 1820.

[23] Klausmann E., Fahrner W. R., Braunig D., Instabilities in Silcon Devices, tome 2. G. Barbottin and A. Vapaille Eds. (Amsterdam, Elsevier Science Publishers B. V., 1989), Chap. 12.

[24] Kirton M. J., Uren M. J., Collins S., Schulz M., Karmann A., Scheffer K., Semicond. Sci. Technol. 4 (1989) 1116. 